\title{
Bidding simulation methods for power suppliers considering forward contract
}

\author{
Heng Feng ${ }^{1,}$, Zhenglin Yang ${ }^{2, b}$ and Yaxian Zheng ${ }^{3, c}$ \\ 1, 2,3 China Electric Power Research Institute (Nanjing), Nanjing 210003, Jiangsu Province, China \\ a915266332@qq.com, byangzhenglin@epri.sgcc.com.cn, zhengyaxian@epri.sgcc.com.cn
}

Keywords: long-term contract; intelligent agent; Bidding simulation methods;

Abstract. A total electricity market should include spot market and forward contract market. The spot market keep balance on electricity, the other is helpful to advance competition and achieve a more efficient market equilibrium price. In this paper, a bidding strategies model based on intelligent agent is used to simulate the behavior of generators. The influence of forward contract on the bidding behavior of generators is considered in the model. The case analysis shows that generator's intelligent agent model established in this paper can well simulate generators of different characteristics; different kinds of long-term contracts have a different influence on bidding behavior of day ahead market.

\section{Introduction}

The goal of reforming electricity industry is for deregulating, introducing the competition to allocate the resources optimally and find maximal social welfare. With the development of China's electricity market, the proportion of market electricity transaction is increasing in the proportion of total sales of electricity. In the competitive electricity market, generation companies can bid through day ahead market, and can also sign a certain proportion of the electricity contract in advance ahead of the spot market, which can avoid the spot market price fluctuation, enhance market liquidity, and reduce the default risk of market participants. The long-term electricity contracts have an influence on the bidding behavior of power suppliers in the day ahead market, the day ahead market trading center is cleared through the bidding of market members. Many generators may avoid the electricity price fluctuation, so they will lock most of the electricity through the contract market. If the cost of generation companies is relatively low, they will bid most of the electricity through the spot market. When electricity market reach a mature stage, most of the basic electricity will trade through the contract market. Everything is still under exploration in the initial operation of the electricity market, but the construction and operation of electricity market is a complex system engineering. The cost of trial and error is huge, so it is necessary to make a forward-looking study to reduce the risk of market model selection. At present, a variety of electricity experimental environments have been established both at domestic and abroad [1-3], which not only require the establishment of market rule and market model, but also have the ability to simulate the decision-making behavior of market participants.

The paper [4] develop an agent-based simulation model of an actual system, which introduce voluntary forward markets mitigating the market power of electricity generating companies by encouraging them to participate in contract forward. It find, in general, that larger companies prefer to exercise market power in the spot market, while smaller companies prefer to contract forward.The paper [5] considers a flexible forward contract implementation in the market environment. A market participant is able to draw electricity from the forward contract and resell it to the spot or retail market. Several stochastic optimization problems are presented for the flexible fomard contract scheduling. A dynamic programming scheme is described for the problem solving. The numerical example demonstrates a procedure for the bidding curve construction, which is useful for making the contract arrangement. This paper [6] shows how volatile pool prices can introduce significant earnings risk to a generating company operating in a competitive power pool. The paper describes how the use of certain financial instruments, "contracts for differences", can help to limit the financial risk, improve the level of earnings for generating companies and stabilize the cost of meeting unpredictable demands. Examples are presented to illustrate what can be done to minimize the risk and to maximize potential 
profits. This paper [7] develops a repeated Cournot game model for two markets, which is presented to try to analyse and predict the generator's risk-averse bidding behaviours in the forward and spot market. The spot price uncertainty, network congestion, multi-period spanning of forward contracts and the operating constraints of generation are taken into account in the model. Numerical testing results show that the introduction of a forward market does encourage market participants to compete more aggressively. It is found that the bidding behaviours are affected by generators' risk tolerance, unit operating constraints, network transfer capability etc. This paper [8] addresses the problem a generating utility faces in order to decide which of its thermal units to start-up and to shutdown before submitting the hourly bids to a day-ahead market based on simple bids. The self-unit commitment model is formulated as a deterministic optimisation problem where the expected profit is maximised using mixedinteger linear programming techniques. This paper [9] develops the premise of constructing mathematical model of power generation company considering the optimal bidding strategies of risk constraints in the day ahead the market on the assumption that the statute requiring power companies to use the market A linear quotation function is used to declare the quotations of each period of the next trading day and the market is carried out separately for each period of time according to the unified liquidation price. This paper [10] develops the premise of constructing mathematical model of power generation company considering the optimal bidding strategies of risk constraints in the day ahead the market on the assumption that the statute requiring power companies to use the market A linear quotation function is used to declare the quotations of each period of the next trading day and the market is carried out separately for each period of time according to the unified liquidation price. This paper [11] develops market - oriented operation of the world's power industry has brought unprecedented market risk to market participants. As an effective The risk management tools and trading means, power forward contracts, including alternative forward contracts that combine options trading ideas, are competing. The power market has been widely used and is developing in the direction similar to futures trading. The article briefly introduces the power market Several forward contract transactions, the risk modeling theory of forward contract trading, and the impact mechanism of forward contract market on the spot market

\section{Theoretical Model}

This paper mainly analyses intelligent agent bidding behavior of power suppliers, which consider forward contracts model, most of the papers put forward the intelligent agent model of forward contracts, but the coordination optimization model of forward contracts and spot market has done a brief construction, its conclusion only certain applicability, and intelligent agent is able to bypass the phenomenon to essence.

With the power industry going from monopoly to competition, the decision-making of market participants will change greatly, especially for generators: the traditional power suppliers only need to arrange the unit output and the unit maintenance plan according to the scheduling, but in the electricity market environment, the generators are based on marginal cost, and utilize the market information to optimize the bidding behavior. However, generators own limited external information at the early stage of electricity market, and many different factors influence bidding of generators in electricity market environment; for the generators themself, their bidding decision have different decision-making preference and risk preference. The above factors increase the uncertainty of bidding behaviors, therefore, the bidding behavior should be considered in many dimensions.

\section{Intelligent agent model}

In order to maximize the benefit, rational generators will continue to learn from the experience of competitive bidding in order to improve the profit level of the next auction. The intelligent agent can use the past historical experience to optimize the follow-up strategy, and characterize the dynamic learning ability of generators; the bidding behavior of generators changes from centralized decision under planning system to decentralized decision in competitive market, intelligent agent can make use 
of limited external information to make decisions autonomously, and interact well with other intelligent agents. Intelligent agent can represent different types of generators through the setting of related factors in the algorithm. Based on the above reasons, this paper uses the intelligent agent method to simulate the bidding behavior of generators.

The intelligent agent model proposed in this paper first needs to establish a reasonable set of bidding strategies for generators, give each element in the bidding strategy space a certain initial probability and propensity coefficient. Then, through the roulette algorithm, the bidding strategy of the generator is continuously selected. After the ISO clearing, a timely return is generated, and the probability and propensity coefficient of each element are updated by immediate return. After several rounds of rotation, the generator will converge on the strategy that will maximize the revenue. In this paper, VRE-learning algorithm is used to simulate the bidding behavior of generators. The algorithm was put forward by Roth and Erev [13] in 1995. The algorithm and the corresponding decision module are presented in document [7].

The update formula for the propensity coefficient in the VRE algorithm is as follows:

$$
q_{t+1}(m)=\left\{\begin{array}{cc}
(1-r) q_{t}(m)+(1-e) \text { profit }_{t} & m=k \\
(1-r) q_{t}(m)+\frac{e \cdot q_{t}(m)}{M} & m \neq k
\end{array}\right.
$$

)

Where $\mathrm{q}_{\mathrm{t}+1}$ : update for propensity coefficient for round $\mathrm{t}+1$; profit $\mathrm{t}_{\mathrm{t}}$ immediate gains for round $\mathrm{t}$; $\mathrm{t}$ :for the bidding round; $\mathrm{m}$ :the policy number selected in a round; M:policy number for policy space; $r$ is a forget factor, weakening the influence of previous experience and increase the impact of new strategies on behavioral tendencies; e is an empirical parameter. The update formula for the selection probability is as follows:

$$
p_{t}(m)=\frac{\exp \left[q_{t}(m) / c\right]}{\sum_{j=1}^{M} \exp \left[q_{t}(j) / c\right]}
$$

Where, $p_{t}(m)$ represents the probability of the $t$ round update of policy $m$; $c$ is the cooling factor, which determines the influence degree of the propensity coefficient on the selection probability, the selection of parameter $\mathrm{c}$ is dynamically adjusted according to the coefficients of each round strategy, as follows:

$$
c_{t}=\frac{k}{M} \sum_{m} q_{t}(m)
$$

Where, $\mathrm{k}$ is a real number greater than 0 , and its value varies in different systems.

\section{Forward contracts bidding model}

In this paper the long-term contract model shares the bidding of day ahead market in accordance with the units the available hours of the units, the part of generation company's electricity is locked in the auction of the day ahead market. The utility function of the day ahead market superpose decomposition of forward contracts before the auction, which can simulate the bidding strategy under the influence of long-term contracts. In this paper, effects of the set of 3 case comparative analysis of long-term contracts for the spot market price bidding of power producers.

\section{Case analysis}

The case in this paper is supported by integrated experimental platform for electricity market which is built by Key Laboratory of State Grid Corporation of China which is electricity market operation technology laboratory. This platform is designed in three dimensions from the electricity transaction 
operation simulation, the market participant behavior simulation and the power grid operation simulation as the overall design, the 5 node system is built on the experimental platform to illustrate the intelligent agent model and learning algorithm used in the electricity market simulation. The network topology is shown in Figure 1, the simulation system has three independent generators and six transmission lines, each generator bids using intelligent agent. The marginal cost data of generating units are shown in Table 1. Because we only consider the day ahead market bidding, the fixed cost according to the average utilization hours allocates the bidding cost in the day ahead market, this paper uses processing method of the [9] model about cost data of generators. In accordance with the corresponding calculation rules, the generator's strategic space is

$$
s=[0.8,0.9,1.1,1.2,1.22,1.28,1.29,1.3,1.31,1.33,1.35,1.38,1.4,1.42,1.44,1.5,1.6,1.7,1.8,1.9] \text {; }
$$

In order to simplify the processing, three generators in this paper use the same policy space, and the intelligent decision module of generators is the same. The intelligent algorithm factor is set as follows: $\mathrm{k}=2, \mathrm{r}=0.09$.

Table. 1 The parameters of generators

\begin{tabular}{lccc}
\hline $\begin{array}{l}\text { generato } \\
\text { rs }\end{array}$ & $\begin{array}{c}\mathrm{a} /(\$ . \\
\left.(\mathrm{MW} . \mathrm{h})^{-1}\right)\end{array}$ & $\begin{array}{c}\mathrm{b} /\left(\$ \mathrm{MW}^{2} \cdot \mathrm{P}_{\mathrm{MAX}} / \mathrm{M}\right. \\
\left.\mathrm{h}^{-1}\right)\end{array}$ & $\mathrm{W}$ \\
\hline 1 & 1.3 & 0.22 & 250 \\
2 & 1.2 & 0.17 & 300 \\
3 & 1.0 & 0.245 & 300
\end{tabular}

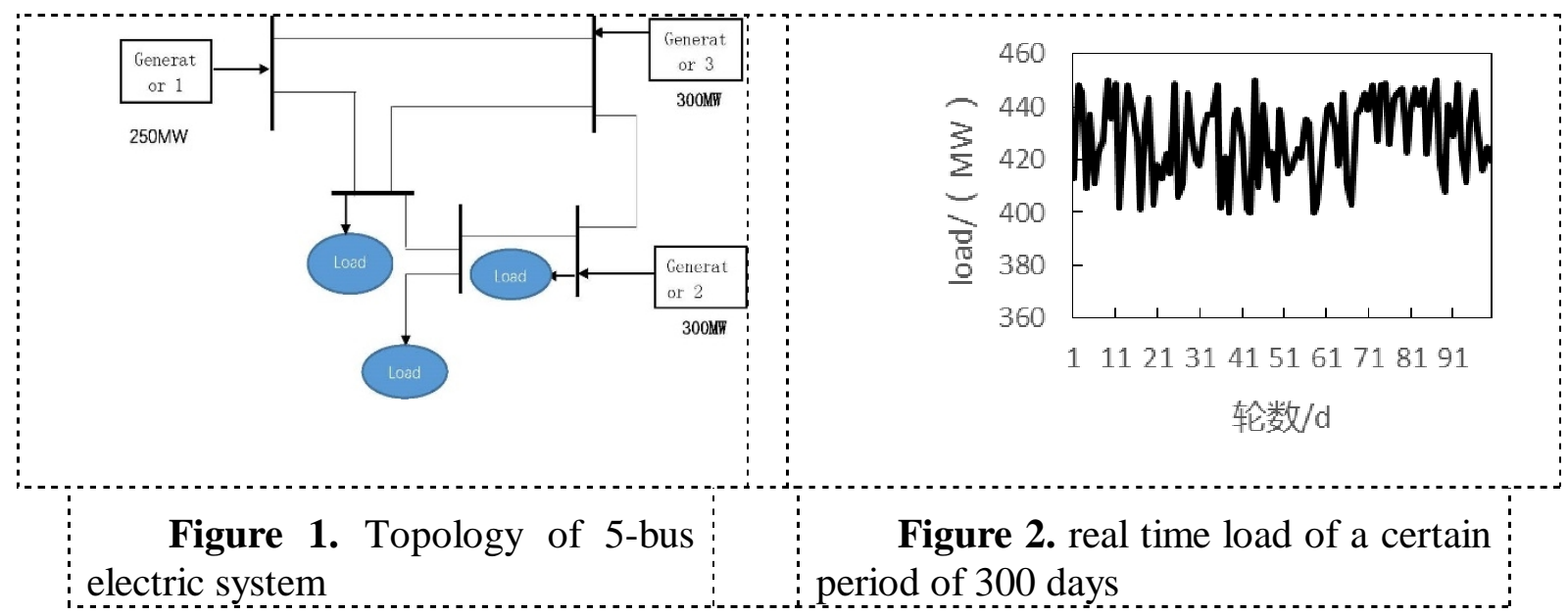

The generator offers only one bid per day, and system load is a period of load during 300 days. Figure 2 is the load data for the selected 100 days period at this time, the average load value was 427 $\mathrm{MW}$, and the variance was $14.6(\mathrm{MW})^{2}$.In the model, the load of this period is set up as random fluctuation, its purpose is to verify that the intelligent agent, which represents the generators, has the learning characteristics under the change of market supply and demand.

Case 1 : generation companies signed long-term contract decomposition to the days before electricity market daily electricity $\mathrm{Q}=300 \mathrm{MW}$, the contract price of $\$ 50$ / $(\mathrm{MWh})$, because of its installed capacity of $300 \mathrm{MW}$, so it's not winning power may be greater than the contract quantity decomposed. The generator's optimal strategy focused on price factor lower, because the power producers by reducing the offer will get more successful power, and lower prices caused by the market clearing price decrease greatly increases the profit of generator, while the power generation will increase the power of the increase, but the total revenue increased more than the increase of total cost of production, so the power chamber of commerce at a low price in order to get more revenue. 


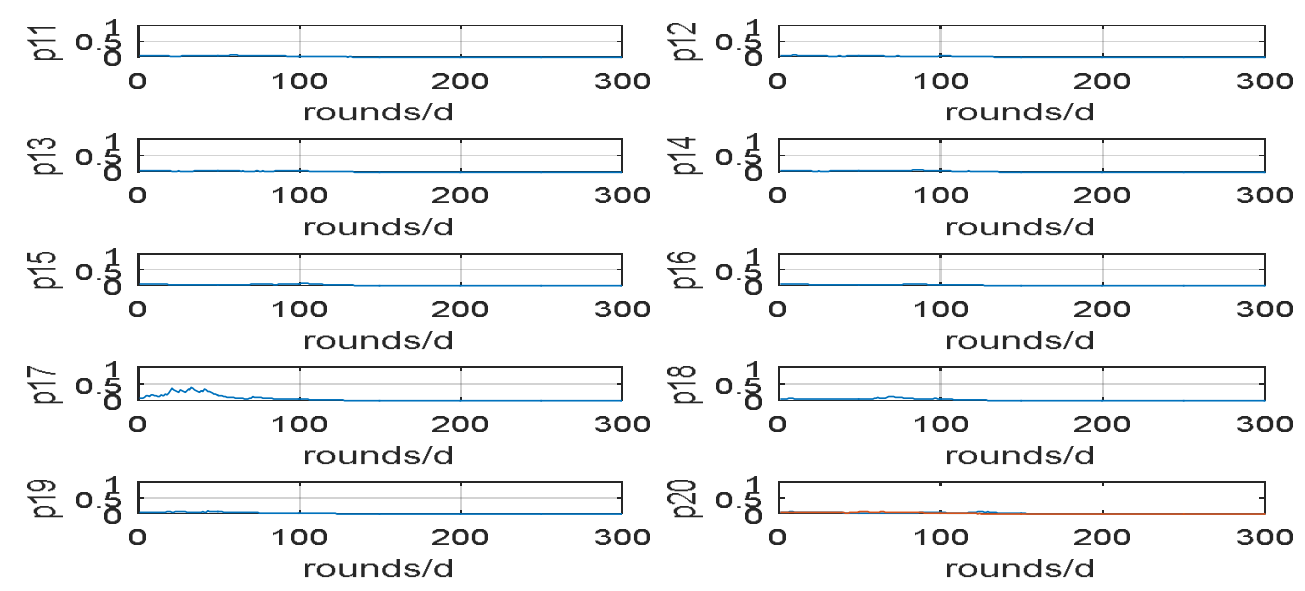

Figure 3. selection of bidding strategies for generators

Case2: generation companies signed long-term contract decomposition to the days before electricity market daily electricity $\mathrm{Q}=100 \mathrm{MW}$, the contract price of $\$ 60$ / (MWh), because of its installed capacity of $300 \mathrm{MW}$, so it's not winning power may be greater than the contract quantity decomposed. The generator's optimal strategy focused on price factor lower, because the power producers by reducing the offer will get more successful power, and lower prices caused by the market clearing price decrease greatly increases the profit of generator, while the power generation will increase the power of the increase, but the total revenue increased more than the increase of total cost of production, so the power chamber of commerce at a low price in order to get more revenue.

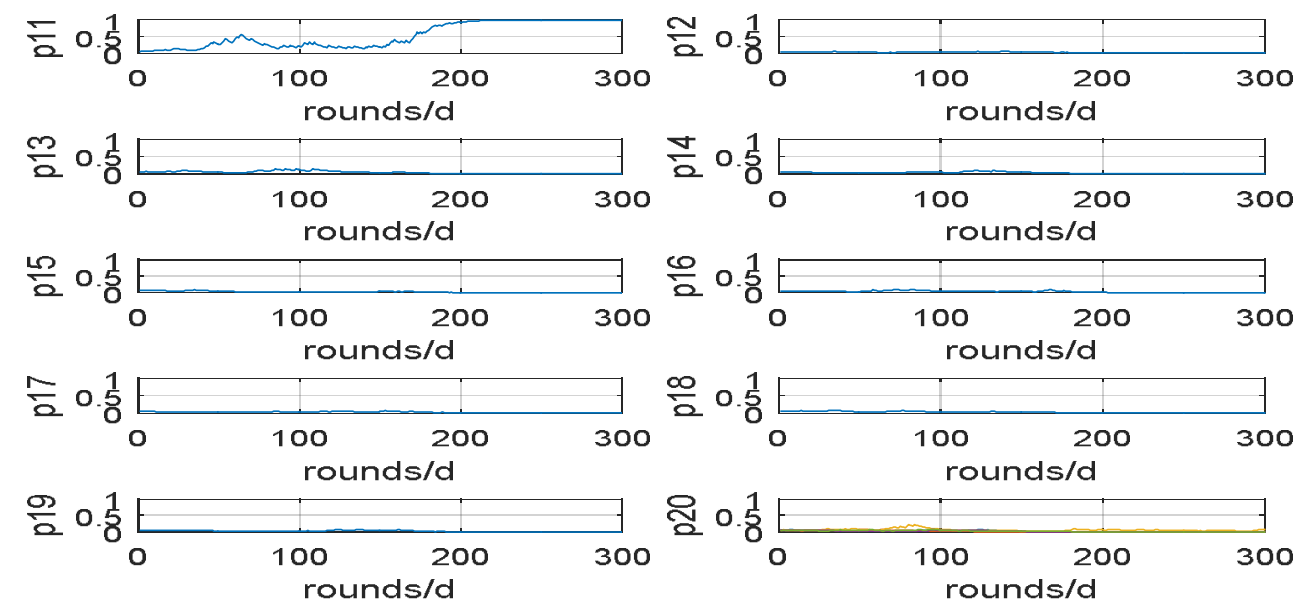

Figure 4. selection of bidding strategies for generators

By calculation, the average income of the generator will be 3976 dollars in 300 days. As can be seen from Figure 3, bidding behavior of generator 2 reaches a relatively stable state after a certain number of study rounds, then proceeding in a small range of fluctuation, this is because the generator bidding based on historical experience of learning have found a more mature strategy. The market reached a balance state, the load change is the only factor affecting generators return volatility at this time.

As can be seen from figure 4, the electricity supplier's bidding earnings are probably in the 20 round or so, reaching a relatively stable state. Figure 3 and Figure 4 comparative analysis: when the decision dependent target parameters I phase at the same time, the learning behavior of power suppliers with different risk preferences are different, figure 8 generation companies in the bidding process soon 
reached a steady state, figure 4 generation after a long time of learning to reach steady state, this is because changes in risk resist generators is not easy to accept external conditions, is sensitive to the fluctuation of income, get some profit will reach maturity, change and risk loving power suppliers receptive environment and returns, after a long time of learning will reach maturity.

\section{Conclusions}

Different types of forward contracts have significant influence on the day ahead market, if power producers signed a part of the price contracts in the contract market, then the case analysis shows that in the days before the auction, bidding strategies in high price factor strategy, power generation companies will use the remaining the bidding capacity for more profit; if the power suppliers signed a long-term contract price in excess before the date of the market, so he in the spot market bidding strategy will tend to be conservative, together with the market price to get more power and lower the report.

\section{Acknowledgements}

This work was supported by the Science and Technology Project of State Grid Corporation of China under Grant (DZN17201600244.)

\section{References}

[1].North M, Conzelmann G, Koritarov V, et al. E-laboratories: agent-based modeling of electricity markets.[J]. Office of Scientific \& Technical Information Technical Reports, 2002.

[2].Bunn D W, Oliveira F S. Agent-based simulation-an application to the new electricity trading arrangements of England and Wales [J]. IEEE Transactions on Evolutionary Computation, 2001, 5(5):493-503.

[3].Sun J, Tesfatsion L. Dynamic Testing of Wholesale Power Market Designs: An Open-Source Agent-Based Framework [J]. Computational Economics, 2007, 30(3):291-327.

[4] Sanchez J J, Bunn D W, Centeno E, et al. Dynamics in Forward and Spot Electricity Markets[J]. IEEE Transactions on Power Systems, 2009, 24(2):582-591.

[5] Palamarchuk S I. Forward contracts for electricity and their correlation with spot markets[C]// Power Tech Conference Proceedings, 2003 IEEE Bologna. IEEE, 2003:6 pp. Vol.1.

[6] Alcock D, Pang C K. Risk management in a power pool[C]// International Conference on Advances in Power System Control, Operation and Management. IET, 2000:557-560 vol.2.

[7] Wu J, Guan X. Risk-averse strategic gaming in forward and spot electricity markets[J]. Iet Generation Transmission \& Distribution, 2012, 6(5):453-462.

[8] Garcia-Gonzalez J, Barquin J. Self-unit commitment of thermal units in a competitive electricity market[C]// Power Engineering Society Summer Meeting. IEEE, 2000:2278-2283 vol. 4.

[9] Wen Fushuan, David A K . Strategic Bidding for Electricity Supply in a Day—ahead Energy

Market Electric Power Systems Research, 2001,59(3)197 206

[10] Zhang Shaohua, Li Yuzeng, Zhou Yongxing. Strategic Bidding of Power Generators in Electricity Market with Contraclsfor Differences. Automation of Electric Power Systems , 2000 , 24(8):15 22

[11] Wen Fushuan , David A K Optimal Bidding Strategies and Modeling of Imperfect Information Among Competitive Generators IEEE T... s on Power Systems . 2001 , 16(1)15 21 